DFTT 95-32

MPI-PhT/95-43

LU TP 95-11

20 May 1995

\title{
The average number of partons per clan in rapidity intervals in parton showers*
}

\author{
A. Giovannini ${ }^{1} \dagger$, S. Lupia ${ }^{2}{ }^{\ddagger}$, R. Ugoccioni ${ }^{3} \S$ \\ 1 Dip. Fisica Teorica and I.N.F.N. - Sezione di Torino, \\ via Giuria 1, I-10125 Torino, Italy \\ 2 Max-Planck-Institut für Physik, Werner-Heisenberg-Institut \\ Föhringer Ring 6, D-80805 München, Germany \\ 3 Dept. of Theoretical Physics, University of Lund \\ Sölvegatan 14 A, S-223 62 Lund, Sweden
}

\begin{abstract}
The dependence of the average number of partons per clan on virtuality and rapidity variables is analytically predicted in the framework of the Generalized Simplified Parton Shower model, based on the idea that clans are genuine elementary subprocesses. The obtained results are found to be qualitatively consistent with experimental trends. This study extends previous results on the behavior of the average number of clans in virtuality and rapidity and shows how important physical quantities can be calculated analytically in a model based on essentials of QCD allowing local violations of the energy-momentum conservation law, still requiring its global validity.
\end{abstract}

${ }^{*}$ Work supported in part by M.U.R.S.T. under Grant 1994

${ }^{\dagger}$ E-mail: giovannini@to.infn.it

${ }^{\ddagger}$ E-mail: lupia@mppmu.mpg.de

$\S$ E-mail: roberto@thep.lu.se 


\section{Introduction}

The first motivation of our search lies in the relevance of clan structure analysis both in theoretical and experimental study of multiparticle production. It is indeed remarkable that clan structure analysis of charged particle Multiplicity Distributions (MD's) in different reactions and at different c.m. energies, in full phase space (fps) and in symmetric rapidity intervals, $\Delta y$, revealed an extraordinary series of new regularities[1], whose interpretation is still in part puzzling. On the theory side, it is to be pointed out how the idea of the production of independent intermediate objects (clans, clusters, strings...) which then decay following a cascading mechanism, firstly overlooked, starting from 1986 became slowly the corner stone of any model of multiparticle production. The second motivation lies in the lack of a sound justification of clan parameters behavior from first principles within a parton shower model, which is the natural framework of the present study of multiparticle production phenomena in view of the application of Generalized Local Parton-Hadron Duality (GLPHD) 2] as hadronization prescription (see for instance [3]).

Along this line, we introduced the Generalized Simplified Parton Shower (GSPS) model, based on essentials of QCD and local weakening of conservation laws, and, in this model, we calculated analytically [4 the average number of clans produced in a given rapidity interval $\Delta y$ by an ancestor parton of maximum allowed virtuality $W, \bar{N}(\Delta y, W)$. It has been found that $\bar{N}(\Delta y, W)$ behavior predicted by the GSPS model is qualitatively consistent with experimental findings in $e^{+} e^{-}$annihilation at hadron level. In particular, $\bar{N}(\Delta y, W)$ grows in rapidity in a way very close to linear and then bends toward a constant value as $\Delta y$ approaches full phase space. $\bar{N}(\Delta y, W)$ shows also an approximate (5\%) energy independence at ancestor energies below $100 \mathrm{GeV}$. This is actually a very slow decrease which is reminiscent of the results obtained in Monte Carlo simulations for single gluon jets [5]. This model shows also the correct growing of $\bar{N}$ (fps, $W$ ) with initial virtuality $W$. In addition to these results, a new regularity for rescaled quantities is predicted: the ratio of $\bar{N}(\Delta y, W)$ to the value in full phase space $\bar{N}(\mathrm{fps}, W)$ is approximately energy independent within a much higher degree of approximation than that previously seen for $\bar{N}(\Delta y, W)$, when it is plotted as a function of rapidity interval rescaled to full phase space.

These trends of the average number of clans in virtuality and rapidity variables are really remarkable and strongly demand to complete the research program announced at the end of our previous work, i.e., to calculate analytically also the average number of partons per clan in the same variables, $\bar{n}_{c}(\Delta y, W)$, and to check its behavior with experimental data. In order to

do that, first we calculate analytically the average number of partons in the 
shower as a function of rapidity interval $\Delta y$ and virtuality $W$; then, by using previous findings 4 in the average number of clans, $\bar{N}(\Delta y, W)$, we determine the average number of partons per clan, $\bar{n}_{c}(\Delta y, W)$.

The plan of the paper goes as follows. In Section 2 clan definition is reexamined in the framework of a two step process in view of the relevance of multiplicity channels 0 and 1 in our analysis. In Section 3 a summary of the GSPS model is presented. In Section 4 the explicit analytical calculation of the average number of partons in the shower and per clan in the GSPS model is performed. Comments on the obtained results are given in Section 5.

\section{Two step processes and clan definition}

As discussed in the Introduction, the main conclusion of experimental analysis of clan structure parameters is that the parton production process within a shower is a two step process: to the initial production of independent intermediate parton sources (clans), it follows in the second step the production of partons inside each clan, whose average number calculation is the main subject of this paper.

Following the standard procedure [6], let us assume to produce in the first step (independently from the second one) $N$ objects $(N=0,1, \ldots)$ with probability $p_{N}$ and generating function

$$
f(z)=\sum_{N=0}^{\infty} p_{N} z^{N}
$$

Each of the $N$ produced objects gives origin in the second step to partons according to the same multiplicity distribution, $q_{n_{i}},\left(n_{i}=0,1 \ldots, \sum_{i=1}^{N} n_{i}=n\right)$, whose generating function is

$$
g(z)=\sum_{n_{i}=0}^{\infty} q_{n_{i}} z^{n_{i}}
$$

The two step nature of the process is summarized in the equation:

$$
F(z) \equiv \sum_{n=0}^{\infty} P_{n} z^{n}=\sum_{N=0}^{\infty} p_{N}[g(z)]^{N}=f[g(z)],
$$

where $P_{n}$ is the probability to produce $n$ partons and $F(z)$ is the corresponding generating function. It should be pointed out that Eq. (3) is not limited to the case in which all produced clans are identical, but it requires only that all clans can be described by the same generating function. Suppose in fact that a clan's $\mathrm{MD}, q_{n_{i}}(\xi)$, depends on a set of parameters, denoted collectively by $\xi$ : 
if for each clan these parameters are independent of the values of other clans' parameters and of the number of clans generated, then it is possible to define an "average clan" whose MD is the MD of a single clan averaged with the probability distribution function $\phi(\xi)$ that a clan is produced with parameters $\xi:$

$$
\tilde{q}_{n_{i}}=\int q_{n_{i}}(\xi) \phi(\xi) d \xi
$$

In this case Eq. (3) would still be valid with the function $g(z)$ generating now the distribution $\tilde{q}_{n_{i}}$. This is the type of average that will be performed in the context of the GSPS model in Section 4.1.

Notice that when $f(z)$ is the generating function of a Poissonian distribution, i.e.:

$$
f(z)=\exp [\bar{N}(z-1)]
$$

it follows:

$$
F(z)=\exp [\bar{N}[g(z)-1]] .
$$

These equations fully define the class of Compound Poisson Distributions (CPD) with all important related properties [7] and, in particular, the clan concept as group of particles of common ancestor [8]. Notice that each clan contains at least one particle in order to be uniquely defined. This fact corresponds to the request that partons' MD inside a clan is shifted, i.e., $q_{0}=0$. This remark is of particular interest for the extension of our discussion on clan parameters from full phase space to rapidity intervals. In fact, while in full phase space we are sure that each clan satisfies the condition $q_{0}=0$, in rapidity intervals one should be careful because if all partons belonging to a clan fall outside the interval $\Delta y$, then $q_{0}(\Delta y) \neq 0$. The problem now is how to define clan concept in this case, i.e., how to redefine clan structure parameters with at least one parton inside each clan in rapidity intervals. The goal for a CPD is obtained by solving the equation:

$$
\exp \left\{\bar{N}(\mathrm{fps}, W)\left[g_{\Delta y}(z)-1\right]\right\}=\exp \left\{\bar{N}^{\prime}(\Delta y, W)\left[g_{\Delta y}^{\prime}(z)-1\right]\right\},
$$

where

$$
g_{\Delta y}(z=0) \equiv q_{0}(\Delta y) \neq 0
$$

and

$$
g_{\Delta y}^{\prime}(z=0) \equiv q_{0}^{\prime}(\Delta y)=0
$$

Notice that the right-hand side of Eq. (7) corresponds to the standard parametrization commonly used in fitting procedures.

From Eq. (7), one has:

$$
\bar{N}^{\prime}(\Delta y, W)=\bar{N}(\mathrm{fps}, W)\left[1-q_{0}(\Delta y)\right]=-\log P_{0}(\Delta y),
$$


$P_{0}(\Delta y)$ being the rapidity gap probability and

$$
g_{\Delta y}^{\prime}(z)=\frac{g_{\Delta y}(z)-q_{0}(\Delta y)}{1-q_{0}(\Delta y)}
$$

the rescaled generating function of partons' MD inside a clan. The average number of partons per clan is given then by:

$$
\bar{n}_{c}^{\prime}(\Delta y, W)=\frac{\bar{n}_{c}(\Delta y, W)}{1-q_{0}(\Delta y)}=\frac{\bar{N}(\mathrm{fps}, W)}{\bar{N}^{\prime}(\Delta y, W)} \bar{n}_{c}(\Delta y, W) .
$$

It should be noticed that $\left[1-q_{0}(\Delta y)\right]$ is the probability that at least one parton belonging to a clan falls in the rapidity interval $\Delta y$, and $\bar{N}^{\prime}(\Delta y, W)$ can then be interpreted as the average number of clans in $\Delta y$. The probability to have $N^{\prime}$ clans in the symmetric rapidity interval $\Delta y, p_{N^{\prime}}(\Delta y, W)$, can be

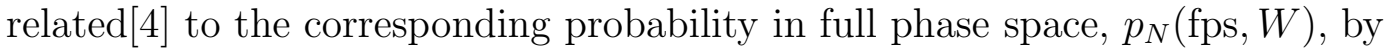
means of the following equation:

$$
p_{N^{\prime}}(\Delta y, W)=\sum_{N=N^{\prime}}^{\infty} \Pi\left(N^{\prime}, \Delta y \mid N, \mathrm{fps}\right) p_{N}(\mathrm{fps}, W),
$$

where $\Pi\left(N^{\prime}, \Delta y \mid N\right.$, fps $)$ is the conditional probability to have $N^{\prime}$ clans in $\Delta y$ when one has $N$ clans in full phase space. Since in [4] clans in full phase space are independently produced, $\Pi\left(N^{\prime}, \Delta y \mid N\right.$, fps $)$ turns out to be in this case a binomial distribution:

$$
\Pi\left(N^{\prime}, \Delta y \mid N, \text { fps }\right)=\left(\begin{array}{c}
N \\
N^{\prime}
\end{array}\right) \pi(\Delta y, W)^{N^{\prime}}[1-\pi(\Delta y, W)]^{N-N^{\prime}},
$$

where $\pi(\Delta y, W)$ is the conditional probability to have one clan in $\Delta y$ when one clan is produced in full phase space.

This result coincides with the previous one (see Eq. (10)); one should simply identify $\pi(\Delta y, W)$ with $\left[1-q_{0}(\Delta y)\right]$, i.e., with the probability that at least one parton in a clan falls in $\Delta y$.

The discussion so far has dealt with a distribution for clans that allows events in which no clans are produced, i.e., empty events. While this may be appropriate at charged hadron level, at parton level it is not because, in order to have a single jet, we must have at least one parton, hence at least one clan (in the following we will refer to this problem as to the "contribution of the ancestor" because it is the ancestor parton which is always present in the jet). In full phase space, if additional clans are again produced according to a Poisson distribution, this contribution leads to the following equation for generating functions:

$$
F_{\mathrm{fps}}(z)=g_{\mathrm{fps}}(z) \exp \left\{\lambda(\mathrm{fps}, W)\left[g_{\mathrm{fps}}(z)-1\right]\right\},
$$


with

$$
\lambda(\mathrm{fps}, W)=\bar{N}(\mathrm{fps}, W)-1
$$

and

$$
g_{\mathrm{fps}}(z=0) \equiv q_{0}(\mathrm{fps})=0 .
$$

Accordingly, in rapidity intervals $\Delta y$, Eqs. (15) and (17) become:

$$
F_{\Delta y}(z)=g_{\Delta y}(z) \exp \left\{\lambda(\mathrm{fps}, W)\left[g_{\Delta y}(z)-1\right]\right\}
$$

with

$$
g_{\Delta y}(z=0) \equiv q_{0}(\Delta y) \neq 0 .
$$

In order to properly define clans in rapidity intervals $\Delta y$, the calculation goes as in the previous scheme without the contribution of the ancestor and the redefinition procedure gives:

$$
g_{\Delta y}(z) \exp \left\{\lambda(\mathrm{fps}, W)\left[g_{\Delta y}(z)-1\right]\right\}=\exp \left\{\lambda^{\prime}(\Delta y, W)\left[g_{\Delta y}^{\prime}(z)-1\right]\right\}
$$

with $g_{\Delta y}^{\prime}(0)=0$.

Notice that the distribution on the r.h.s. must now allow for intervals empty of partons, hence it is not shifted, and also that the average number of Poisson distributed objects from this form results to be $\lambda^{\prime}(\Delta y, W)$. It follows:

$$
\begin{gathered}
\lambda^{\prime}(\Delta y, W)=\lambda(\mathrm{fps}, W)\left[1-q_{0}(\Delta y)\right]-\log q_{0}(\Delta y)=-\log P_{0}(\Delta y), \\
g_{\Delta y}^{\prime}(z)=1+\frac{\lambda(\mathrm{fps}, W)\left[g_{\Delta y}(z)-1\right]+\log g_{\Delta y}(z)}{\lambda^{\prime}(\Delta y, W)}
\end{gathered}
$$

and

$$
\bar{n}_{c}^{\prime}(\Delta y, W)=\frac{\lambda(\mathrm{fps}, W)+1}{\lambda^{\prime}(\Delta y, W)} \bar{n}_{c}(\Delta y, W) .
$$

Equations (21), (22) and (23) should be compared with Eqs. (10), (11) and (12), where the contribution of the ancestor is not taken into account.

It should be pointed out that the above procedure is correct only if the rescaled generating function of partons' MD inside a clan, $g_{\Delta y}^{\prime}(z)$, is properly defined, i.e., if

$$
g^{\prime}(z=1)=1
$$

and

$$
q_{n}^{\prime}(\Delta y) \propto\left(\frac{d^{n} g_{\Delta y}^{\prime}(z)}{d z^{n}}\right)_{z=0} \geq 0 .
$$

Being $g(z=1)=1$, thanks to Eq. (22), the first condition is trivially fulfilled; the second condition, Eq. (25), can be reformulated as follows:

$$
q_{n}^{\prime}(\Delta y)=\frac{\lambda(\mathrm{fps}, W)}{\lambda^{\prime}(\Delta y, W)} q_{n}(\Delta y)+\frac{1}{\lambda^{\prime}(\Delta y, W)}\left(\frac{d^{n} \log g_{\Delta y}(z)}{d z^{n}}\right)_{z=0} \geq 0
$$


and it is fulfilled if

$$
\left(\frac{d^{n} \log g_{\Delta y}(z)}{d z^{n}}\right)_{z=0} \geq 0 \quad \forall n
$$

i.e., if $\log g_{\Delta y}(z)$ is a generating function up to a normalization factor. Being $\log \left(g_{\Delta y}(z) / q_{0}(\Delta y)\right)$ the generating function of combinants associated in $\Delta y$ with partons' MD inside a clan[7], $g_{\Delta y}(z)$, the validity of Eq. (27) for all $n$ implies that combinants are positive definite, i.e., that the corresponding MD inside a clan is also a CPD [9].

Notice that now the binomial convolution of Eq. (13), applied in [4, gives a result different from Eq. (21):

$$
\bar{N}^{\prime \prime}(\Delta y, W)=\left[1-q_{0}(\Delta y)\right] \bar{N}(\mathrm{fps}, W) \neq \lambda^{\prime}(\Delta y, W) ;
$$

it is $\bar{N}^{\prime \prime}(\Delta y, W)$ which should properly be identified as the average number of clans that produce at least one parton in the interval $\Delta y$. Thus in the present case in which at least one clan is always produced one cannot simply identify the parameter which appears in a CPD description of the MD in a rapidity interval with the average number of clans actually contributing to that interval: the former, given in Eq. (21) diverges when the interval $\Delta y$ goes to fps; the latter, given in Eq. (28), tends to $\bar{N}(\mathrm{fps}, W)$ in the same limit. This point does not arise in the experimental measure of charged hadron MD for the simple reason that events with no charged particles in full phase space are allowed (although they are not measured). The detailed analysis we are carrying out in this paper has brought this problem to the surface: earlier work on the MD at parton level 10 has by-passed this difficulty by neglecting multiplicities 0 and 1 in full phase space: this was indeed justified as the interest was mainly in small rapidity intervals and, more important, the concept of clans was still a statistical one. But in making the step and treating clans as genuine subprocesses, this resolution is no longer appropriate; the consequences of this fact will be discussed in Section 5 together with our results.

Having clarified this important point on clan definition in a two step process, we can now proceed to calculate in the framework of the GSPS model the average number of particles inside a clan as a function of rapidity and virtuality variables, i.e.:

$$
\bar{n}_{c}(\Delta y, W)=\left.\frac{d g_{\Delta y}(z)}{d z}\right|_{z=1}
$$

It should be clear that clan parameters used in experimental analysis differ from those calculated in the GSPS model via Eq. (29). In fact, in experimental 
analysis, one determines $g_{\Delta y}^{\prime}(z)$ (Eq. (22)) and, accordingly,

$$
\bar{n}_{c}^{\prime}(\Delta y, W)=\left.\frac{d g_{\Delta y}^{\prime}(z)}{d z}\right|_{z=1} .
$$

The primed quantities of Eq. (30) are of course linked to the unprimed ones of Eq. (29) by Eqs. (21) - (23).

It is to be pointed out that the average number of particles in a given rapidity interval $\Delta y, \bar{n}(\Delta y, W)$, can be determined both before and after the redefinition process. One has:

$$
\bar{n}(\Delta y, W)=\bar{N}(\mathrm{fps}, W) \bar{n}_{c}(\Delta y, W)
$$

and

$$
\bar{n}(\Delta y, W)=\lambda^{\prime}(\Delta y, W) \bar{n}_{c}^{\prime}(\Delta y, W)
$$

respectively; notice that, as expected, $\bar{n}(\Delta y, W)$ is independent of the redefinition procedure.

\section{The GSPS model: a summary}

The description of a parton shower initiated by an ancestor parton of virtuality $Q$ splitting into two partons of virtualities $Q_{0}$ and $Q_{1}$ in terms of virtuality and rapidity variables is usually given within LLA QCD evolution equations 11 by:

$$
\frac{d P_{Q \rightarrow Q_{0} Q_{1}}}{d t}=\frac{\alpha_{s}(t)}{2 \pi} \int_{z_{\min }}^{z_{\max }} P_{Q \rightarrow Q_{0} Q_{1}}\left(z_{0}\right) d z_{0},
$$

where

$$
\alpha_{s}(t)=\frac{12 \pi}{\left(11 N_{c}-2 N_{f}\right) t}, \quad t=\log \frac{Q^{2}}{\Lambda^{2}} .
$$

$N_{c}$ and $N_{f}$ are the number of colors and flavors respectively and $\Lambda$ is the QCD scale.

$P_{Q \rightarrow Q_{0} Q_{1}}(z)$ is the splitting kernel and $z_{0}$ is the energy fraction carried away by the parton of virtuality $Q_{0}$. The virtuality at which the parent parton splits as well as the energy fraction carried away by the daughter partons is determined at each splitting. The main problem in the standard approach concerns how to take care of energy-momentum conservation law $\left(z_{0}+z_{1}=\right.$ 1). In the GSPS model, a different approach is followed. The branching process is described here in fact in terms of finite splitting functions 12 and this procedure allows to fix kinematical limits on the energy fraction $z_{0}$ by two-body kinematics 10 . 
For the virtuality dependence of the splitting function, a factorized form inspired to QCD and suitably normalized by a Sudakov form factor term has been chosen:

$p_{A}\left(Q_{0} \mid Q\right) d Q_{0}=p_{0}^{A}(Q) C_{A}(Q) d Q_{0}=C_{A}(Q) \frac{d}{d Q_{0}}\left(\frac{1}{C_{A}\left(Q_{0}\right)}\right) d Q_{0}=d\left(\frac{\log Q_{0}}{\log Q}\right)^{A}$,

where $A$ at this level of investigation is the only free parameter of the model and controls the length of the cascade.

We include next clans as independently produced intermediate parton sources and modify accordingly the kinematics of the process by allowing local violations of the energy-momentum conservation law, still requiring its global validity, i.e., offspring partons of virtualities $Q_{i}$ can fluctuate according to:

$$
Q_{0}+Q_{1} \not \leq Q \quad, \quad 1 \mathrm{GeV} \leq Q_{i} \leq Q \quad[\mathrm{i}=0,1] .
$$

Under this assumption, the joint probability density $\mathcal{P}\left(Q_{0} Q_{1} \mid Q\right)$ becomes factorized:

$$
\mathcal{P}\left(Q_{0}, Q_{1} \mid Q\right) d Q_{0} d Q_{1}=p_{A}\left(Q_{0} \mid Q\right) d Q_{0} p_{A}\left(Q_{1} \mid Q\right) d Q_{1} .
$$

Each parton emission is therefore independent in virtuality. Of course, constraints on the energy fraction carried away by daughter partons are also no longer valid, i.e., $z_{0}+z_{1} \neq 1$, and kinematical bounds in rapidity are loosened:

$$
\left|y_{i}-y\right| \leq \log \frac{Q}{Q_{i}} \quad[\mathrm{i}=0,1] .
$$

This new condition modifies the splitting kernel in $z$ which now is decoupled:

$$
P\left(z_{0}, z_{1}\right) d z_{0} d z_{1} \propto \frac{d z_{0}}{z_{0}} \frac{d z_{1}}{z_{1}} .
$$

By combining Eqs. (37) and (39), one sees that the total bi-dimensional splitting function is also decoupled and corresponds to two independent emissions of a single parton, i.e., in order to describe the production process, it is enough to follow just one branch of the shower evolution. Eq. (39) can be rewritten in terms of rapidity variables for each branch:

$$
Y\left(\left|y_{i}-y_{i+1}\right|, Q_{i}, Q_{i+1}\right) d y_{i} \propto \frac{d z_{i}}{z_{i}}=d y_{i},
$$

where $Y\left(\left|y_{i}-y_{i+1}\right|, Q_{i}, Q_{i+1}\right)$ is the probability that a parent parton with rapidity $y_{i+1}$ generates in a single step a parton of rapidity $y_{i}$, being their 
virtualities $Q_{i+1}$ and $Q_{i}$ respectively. When properly normalized, Eq. (40) turns out to be:

$$
Y\left(\left|y_{i}-y_{i+1}\right|, Q_{i}, Q_{i+1}\right) d y_{i}=\frac{d y_{i}}{2 \log \left(\frac{Q_{i+1}}{Q_{i}}\right)} \theta\left(\log \left(Q_{i+1} / Q_{i}\right)-\left|y_{i}-y_{i+1}\right|\right) .
$$

By identifying now in each splitting one of the produced partons as the ancestor parton of a clan, one obtains that clans' production process is Markoffian and therefore clans are independently produced; notice that this a property of the model and not an assumption, as discussed in [4]. The explicit calculation of clans' MD in a parton shower originated by an ancestor parton of virtuality $W$ gives in fact a shifted poissonian distribution with average number

$$
\bar{N}(\text { fps, } W)=1+A \log \left(\frac{\log W}{\log 2}\right) \text {. }
$$

Results of this scheme of parton shower evolution have been summarized in the Introduction.

We concluded that the observed qualitative agreement of the predictions of the model with experimental behavior for the average number of clans in rapidity intervals supports our idea that clans can be considered independent intermediate parton sources, i.e., that local fluctuations in the virtualities of produced partons can occur along the shower. This fact is quite acceptable since energy-momentum conservation laws are expected to weaken in limited rapidity intervals whereas their rôle is surely fundamental in full phase space.

Finally notice also that the approximation used in the GSPS model closely resembles that used in the dipole model[13], where two subsequent dipole emissions are needed in order to describe a full branching. Furthermore, the kinematical structure of the model is similar. The main difference among the two models lies in the description of the shower evolution: in fact, it is based on finite splitting functions in the GSPS model, whereas it follows the standard approach with elementary splitting kernels in the dipole model.

\section{The average number of partons per clan in the GSPS model}

\subsection{The structure of the calculation}

In order to study the average number of clans in the rapidity interval $\Delta y$, $\bar{N}(\Delta y, W)$, in [4] we limited our discussion to the first step of parton shower evolution in the GSPS model. It is clear that if one wants to calculate the 
average number of partons per clan in the same interval, $\bar{n}_{c}(\Delta y, W)$, one has to analyze the second step of parton shower evolution, i.e., to study the production of partons inside clans. In order to do that, inspired by the criterium of simplicity and previous findings 10, 14, we decided to maintain inside a clan the structure of the model seen in the first step. The only difference (see Eq. (35)) lies in the introduction of a new parameter, $a$, controlling the length of the cascade inside a clan, in the expression of the probability that a parton of virtuality $Q$ emits a daughter parton in the virtuality range $\left[Q_{0}, Q_{0}+d Q_{0}\right]$, i.e.,

$$
p_{a}\left(Q_{0} \mid Q\right) d Q_{0}=d\left(\frac{\log Q_{0}}{\log Q}\right)^{a} .
$$

The model becomes of course a two-parameter model ( $A$ and $a$ controlling the length of the cascade in step 1 and 2 respectively).

Let us now consider a clan splitting at virtuality $Q$ and study the evolution equation for the generating function of partons' MD in full phase space inside a clan, $g_{\mathrm{fps}}(z, Q)$, which, according to the pure-birth structure of the model, turns out to be:

$$
\frac{d g_{\mathrm{fps}}(z, Q)}{d Q}=p_{a}(Q \mid Q)\left[g_{\mathrm{fps}}^{2}(z, Q)-g_{\mathrm{fps}}(z, Q)\right]
$$

where $p_{a}(Q \mid Q)$, from Eq. (43), is given by

$$
p_{a}(Q \mid Q)=\frac{a}{Q \log Q} .
$$

Notice that, as expected, Eq. (44) corresponds to the evolution equation for the generating function of gluons' MD in a gluon jet in Leading Log Approximation of QCD with fixed cutoff infrared regularization [15], as well as to the limit of the Simplified Parton Shower model when one weakens conservation laws according to Eq. (36) [10]. Of course Eq. (44) concerns now partons' MD inside a single clan.

The solution of Eq. (44) is

$$
\begin{aligned}
g_{\mathrm{fps}}(z, Q) & =\frac{z}{1+(\bar{n}(Q)-1)(1-z)} & & Q \geq 2 \mathrm{GeV} ; \\
& = & & Q<2 \mathrm{GeV},
\end{aligned}
$$

where

$$
\bar{n}(Q)=e^{\lambda_{a}(Q)}, \quad \lambda_{a}(Q)=\int_{2}^{Q} p_{a}\left(Q^{\prime} \mid Q^{\prime}\right) d Q^{\prime} .
$$

The solutions correspond to a shifted geometric distribution ( $\mathrm{Q} \geq 2 \mathrm{GeV})$ and to a clan with only one parton $(\mathrm{Q}<2 \mathrm{GeV})$. The bound $2 \mathrm{GeV}$ is a 
consequence of the fact that in the GSPS model the virtuality cutoff is fixed at $1 \mathrm{GeV}$ (a parton with virtuality $Q<2 \mathrm{GeV}$ cannot split any further by assumption). Notice that this finding agrees with the clan model discussed in [14], where logarithmic MD for partons inside average clans is interpreted as the result of an average over geometrically distributed single clans of different multiplicity.

Equation (46) answers our first question and gives the generating function of partons' MD inside a single clan in full phase space. In order to match our theoretical calculations with experiments, one should introduce the rapidity dependence in the model and average single clan properties over clans of different rapidity and virtuality. According to our idea that single clans are independent intermediate parton (gluon) sources acting both in virtuality and rapidity spaces, and not only statistical objects as in the original clan model, we decided first to extend the model defined in full phase space to rapidity intervals and then to average these results on single clans' MD's.

Following the discussion contained in Section 2, we calculate the generating function of partons' MD inside a clan in rapidity intervals $\Delta y$ through a binomial convolution on the corresponding generating function in full phase space:

$$
\begin{aligned}
g_{\Delta y}(z, Q, y) & =\frac{1+(z-1) \pi_{a}(\Delta y, Q, y)}{1+\pi_{a}(\Delta y, Q, y)(\bar{n}(Q)-1)(1-z)} \quad Q \geq 2 \mathrm{GeV} ; \\
& =1+(z-1) \pi_{a}(\Delta y, Q, y) \quad Q<2 \mathrm{GeV}
\end{aligned}
$$

where $\pi_{a}(\Delta y, Q, y)$ is the probability that a clan of initial virtuality $Q$ and rapidity $y$ produces a daughter parton inside the interval $\Delta y$. Notice that

$$
\pi_{a}(\Delta y, Q, y)=\int_{\Delta y} \rho\left(y_{f} \mid Q y\right) d y_{f}
$$

$\rho\left(y_{f} \mid Q y\right)$ is the conditional probability that a final parton produced by a clan of given $Q$ and $y$ has rapidity $y_{f}$.

We have now extended formally the generating function of partons' MD inside a clan from full phase space to rapidity intervals for a single clan of known initial virtuality $Q$ and rapidity $y$. It is clear that in this model a single event contains many clans of different initial parameters, while at experimental

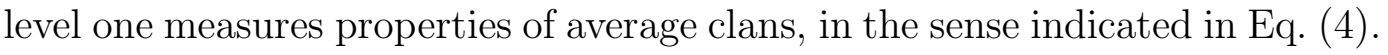
Accordingly, we proceed now to average Eq. (48) over $Q$ and $y$. It follows that:

$$
\bar{g}_{\Delta y}(z, W)=\int_{1}^{W} d Q \int_{-\infty}^{\infty} d y g_{\Delta y}(z, Q, y) \sigma(Q y \mid W)
$$

where $\bar{g}_{\Delta y}(z, W)$ is the generating function of partons' MD in the interval $\Delta y$ for an average clan generated in a shower of virtuality $W ; \sigma(Q y \mid W)$ is the 
probability that a parton of maximum allowed virtuality $W$ produces a clan of virtuality $Q$ and rapidity $y$ and $g_{\Delta y}(z, Q y)$ is given by Eq. (48).

By deriving then Eq. (50) with respect to $z$ and choosing $z=1$, one gets the average number of partons in an average clan generated in a shower of virtuality $W$ in the rapidity interval $\Delta y$, i.e.:

$$
\bar{n}_{c}(\Delta y, W)=\int_{1}^{W} d Q \int_{-\infty}^{\infty} d y \bar{n}_{c}(\Delta y, Q, y) \sigma(Q, y \mid W),
$$

where $\bar{n}_{c}(\Delta y, Q, y)$ is the parton average multiplicity in a rapidity interval $\Delta y$ for a single clan of given $Q$ and $y$, and is related to $\bar{n}_{c}(\mathrm{fps}, Q, y)$ as follows:

$$
\bar{n}_{c}(\Delta y, Q, y)=\pi_{a}(\Delta y, Q, y) \bar{n}_{c}(\mathrm{fps}, Q, y)=\pi_{a}(\Delta y, Q, y) e^{\lambda(Q)} .
$$

Equation (51) formally solves our main problem; the final analytical expression for $\bar{n}_{c}(\Delta y, W)$ results after having determined explicitly the two factors $\bar{n}_{c}(\Delta y, Q, y)$ and $\sigma(Q, y \mid W)$. Notice that the integration domain in $y$ is determined by kinematical constraints in $\sigma(Q, y \mid W)$, which are explicitly discussed in the following Subsection.

In Subsections 4.2 and 4.3 , we determine $\sigma(Q, y \mid W)$ and $\bar{n}_{c}(\Delta y, Q, y)$ respectively, whereas in Subsection 4.4 we perform the final integration over $Q$ and $y$ according to Eq. (51).

\subsection{The probability to produce a clan of given virtuality $Q$ and rapidity $y, \sigma(Q, y \mid W)$}

The weight function $\sigma(Q, y \mid W)$ we are looking for coincides with the bidimensional clan density in virtuality $Q$ and rapidity $y$ for a shower originated by a parton of maximum allowed virtuality $W, p_{\Sigma}(Q, y \mid W)$, up to the normalization factor $\bar{N}($ fps, $W$ ):

$$
\sigma(Q, y \mid W)=\frac{p_{\Sigma}(Q, y \mid W)}{\bar{N}(\mathrm{fps}, W)} .
$$

Notice that $p_{\Sigma}(Q, y \mid W)$ by definition depends on the first step of the production process and therefore the expression of $\sigma(Q, y \mid W)$ is expected to contain the parameter $A$ only.

Following the procedure used in [4], where we calculated the clan density in rapidity, we define here $p_{\Sigma}(Q, y \mid W)$ as:

$$
p_{\Sigma}(Q, y \mid W)=\sum_{N=1}^{\infty} p_{N}(Q, y \mid W)
$$


where $p_{N}(Q, y \mid W)$ is the probability that a parton of maximum allowed virtuality $W$ generates a clan of virtuality $Q$ and rapidity $y$ after $N$ steps.

The explicit formula for $p_{N}(Q, y \mid W)$ in the GSPS model is given by:

$$
\begin{array}{cl}
p_{N}(Q y \mid W) & = \\
\int_{\max \{2, Q\}}^{W} & d Q_{N-1} p_{A}\left(Q_{N-1} \mid W\right) \int_{-\infty}^{\infty} d y_{N-1} \delta\left(y_{N-1}-\tanh ^{-1} \sqrt{1-\left(Q_{N-1} / W\right)^{2}}\right) \\
\int_{\max \{2, Q\}}^{Q_{N-1}} & d Q_{N-2} p_{A}\left(Q_{N-1} \mid Q_{N-2}\right) \int_{-\infty}^{\infty} d y_{N-2} Y\left(\left|y_{N-2}-y_{N-1}\right|, Q_{N-2}, Q_{N-1}\right) \\
\ldots & \\
\int_{\max \{2, Q\}}^{Q_{2}} & d Q_{1} p_{A}\left(Q_{1} \mid Q_{2}\right) \int_{-\infty}^{\infty} d y_{1} Y\left(\left|y_{1}-y_{2}\right|, Q_{1}, Q_{2}\right) \\
p_{A}\left(Q \mid Q_{1}\right) & Y\left(\left|y-y_{1}\right|, Q, Q_{1}\right) .
\end{array}
$$

Following the GSPS model prescriptions, $Y\left(\left|y_{i}-y_{i+1}\right|, Q_{i}, Q_{i+1}\right)$ can be approximated by a gaussian function, i.e.:

$$
Y\left(\left|y_{i}-y_{i+1}\right|, Q_{i}, Q_{i+1}\right)=\frac{1}{\sqrt{2 \pi \log \left(\frac{Q_{i+1}}{Q_{i}}\right)}} \exp \left(-\frac{\left|y_{i}-y_{i+1}\right|^{2}}{2 \log \left(\frac{Q_{i+1}}{Q_{i}}\right)}\right) \text {. }
$$

This approximation allows to perform the integration over all rapidity variables with the exception of $y_{N-1}$. The integrations on the corresponding virtuality variables can be done by using the symmetry properties of the integrand. One gets:

$$
\begin{aligned}
& p_{N}(Q y \mid W)= \\
& \int_{Q}^{W} d Q_{N-1} p_{A}\left(Q_{N-1} \mid W\right) \int_{-\infty}^{\infty} d y_{N-1} \delta\left(y_{N-1}-\tanh ^{-1} \sqrt{1-\left(Q_{N-1} / W\right)^{2}}\right) \\
& p_{A}\left(Q \mid Q_{N-1}\right) Y\left(\left|y-y_{N-1}\right|, Q, Q_{N-1}\right) \frac{\left[\lambda_{A}\left(Q_{N-1}\right)-\lambda_{A}(Q)\right]^{N-2}}{(N-2) !} \\
& \text { for } Q \geq 2 \mathrm{GeV}
\end{aligned}
$$

and

$$
\begin{aligned}
& p_{N}(Q y \mid W)= \\
& \int_{2}^{W} d Q_{N-1} p_{A}\left(Q_{N-1} \mid W\right) \int_{-\infty}^{\infty} d y_{N-1} \delta\left(y_{N-1}-\tanh ^{-1} \sqrt{1-\left(Q_{N-1} / W\right)^{2}}\right) \\
& p_{A}\left(Q \mid Q_{N-1}\right) Y\left(\left|y-y_{N-1}\right|, Q, Q_{N-1}\right) \frac{\left[\lambda_{A}\left(Q_{N-1}\right)\right]^{N-2}}{(N-2) !} \\
& \text { for } Q<2 \mathrm{GeV} .
\end{aligned}
$$


Notice that the last integration over $y_{N-1}$ needs not to be performed by using the gaussian approximation; in fact, the integration can be done with $Y\left(\mid y_{i}-\right.$ $\left.y_{i+1} \mid, Q_{i}, Q_{i+1}\right)$ given in its original form (Eq. (41)). This fact restores global conservation laws in rapidity space.

By using Eq. (35) for $p_{A}\left(Q_{0} \mid Q\right)$ and Eq. (47) for $\lambda_{A}(Q)$, Eq. (54) leads to the following expression for $p_{\Sigma}(Q, y \mid W)$ :

$$
\begin{aligned}
p_{\Sigma}(Q y \mid W)= & p_{0}^{A}(Q) C_{A}(W) \int_{Q}^{W} d Q_{N-1} p_{0}^{A}\left(Q_{N-1}\right) C_{A}(Q) \\
& \int_{-\infty}^{\infty} d y_{N-1} \delta\left(y_{N-1}-\tanh ^{-1} \sqrt{1-\left(Q_{N-1} / W\right)^{2}}\right) Y\left(\left|y-y_{N-1}\right|, Q, Q_{N-1}\right) \\
+ & p_{A}(Q \mid W) \delta\left(y-\tanh ^{-1} \sqrt{1-(Q / W)^{2}}\right) \\
& \text { for } Q \geq 2 \mathrm{GeV}
\end{aligned}
$$

and

$$
\begin{aligned}
p_{\Sigma}(Q y \mid W)= & p_{0}^{A}(Q) C_{A}(W) \int_{2}^{W} d Q_{N-1} p_{0}^{A}\left(Q_{N-1}\right) C_{A}(2) \\
& \int_{-\infty}^{\infty} d y_{N-1} \delta\left(y_{N-1}-\tanh ^{-1} \sqrt{1-\left(Q_{N-1} / W\right)^{2}}\right) Y\left(\left|y-y_{N-1}\right|, Q, Q_{N-1}\right) \\
+ & p_{A}(Q \mid W) \delta\left(y-\tanh ^{-1} \sqrt{1-(Q / W)^{2}}\right) \\
& \text { for } Q<2 \mathrm{GeV} .
\end{aligned}
$$

By integrating over $y_{N-1}$, one has:

$$
\begin{aligned}
p_{\Sigma}(Q y \mid W)= & p_{A}(Q \mid W) C_{A}(\max (2, Q)) \int_{\max (2, Q)}^{W} d Q_{N-1} \frac{p_{0}^{A}\left(Q_{N-1}\right)}{2 \log Q_{N-1} / Q} \\
& \theta\left(\log Q_{N-1} / Q-\left|y-\tanh ^{-1} \sqrt{1-\left(Q_{N-1} / W\right)^{2}}\right|\right) \\
+ & p_{A}(Q \mid W) \delta\left(y-\tanh ^{-1} \sqrt{1-\left(Q_{N-1} / W\right)^{2}}\right) .
\end{aligned}
$$

Equation (61) in the approximation

$$
\tanh ^{-1} \sqrt{1-\left(Q_{N-1} / W\right)^{2}} \simeq \log (2 W)-\log Q_{N-1}
$$

gives:

$$
\begin{aligned}
p_{\Sigma}(Q y \mid W) & =p_{A}(Q \mid W) C_{A}(Q) \int_{1 / 2(\log 2 W-y+\log Q}^{\log W} d\left(\log Q^{\prime}\right) Q^{\prime} \frac{p_{0}^{A}\left(Q^{\prime}\right)}{2 \log Q^{\prime} / Q} \\
& +\quad p_{A}(Q \mid W) \delta\left(y-\tanh ^{-1} \sqrt{1-\left(Q^{\prime} / W\right)^{2}}\right) \\
\text { for } \quad Q \geq 2 \mathrm{GeV} &
\end{aligned}
$$


and

$$
\begin{aligned}
p_{\Sigma}(Q y \mid W)= & p_{A}(Q \mid W) C_{A}(2) \\
& \int_{\max (2, Q)}^{W} d Q^{\prime} \frac{p_{0}^{A}\left(Q^{\prime}\right)}{2 \log Q^{\prime} / Q} \theta\left(\log Q^{\prime} / Q-\left|y-\tanh ^{-1} \sqrt{1-\left(Q^{\prime} / W\right)^{2}}\right|\right) \\
+ & p_{A}(Q \mid W) \delta\left(y-\tanh ^{-1} \sqrt{1-\left(Q^{\prime} / W\right)^{2}}\right) \\
& \text { for } Q<2 \mathrm{GeV} .
\end{aligned}
$$

The region where $p_{\Sigma}(Q y \mid W)$ is different from zero, according to the just mentioned approximation, is given by

$$
-\log \frac{W}{2 Q} \leq y \leq \log \frac{2 W}{Q} .
$$

These results allow to calculate $\sigma(Q, y \mid W)$ from Eq. (53).

\subsection{The average number of partons in $\Delta y$ inside a single clan, $\bar{n}_{c}(\Delta y, Q, y)$}

In view of Eqs. (49) and (52), one has now to calculate the conditional probability that a final parton produced by a clan of given virtuality $Q$ and rapidity $y$ has rapidity $y_{f}, \rho\left(y_{f} \mid Q y\right)$. This calculation can be performed in terms of the probability that a clan of given virtuality $Q$ and rapidity $y$ produces a final parton of rapidity $y_{f}$ after $N$ steps, $r_{N}\left(y_{f} \mid Q y\right)$. It follows:

$$
\rho\left(y_{f} \mid Q y\right)=\sum_{N=1}^{\infty} r_{N}\left(y_{f} \mid Q y\right) \text {. }
$$

Notice that $r_{N}\left(y_{f} \mid Q y\right)$ is obtained by integrating $r_{N}\left(Q_{f} y_{f} \mid Q y\right)$ over the virtuality of the final parton, $Q_{f}$ in the interval $1 \mathrm{GeV} \div 2 \mathrm{GeV}$ :

$$
r_{N}\left(y_{f} \mid Q y\right) \equiv \int_{1}^{2} d Q_{f} r_{N}\left(Q_{f} y_{f} \mid Q y\right) \text {. }
$$

$r_{N}\left(Q_{f} y_{f} \mid Q y\right)$ is in fact the probability that a final parton of virtuality $Q_{f}$ and rapidity $y_{f}$ is generated by a clan of virtuality $Q$ and rapidity $y$ after $N$ steps. The problem is now how to calculate $r_{N}\left(Q_{f} y_{f} \mid Q y\right)$. This calculation can be performed in the GSPS model; in fact, being

$$
\begin{aligned}
r_{N}\left(Q_{f} y_{f} \mid Q y\right)= & \int_{2}^{Q} d Q_{N-1} p_{a}\left(Q_{N-1} \mid Q\right) \int_{-\infty}^{\infty} d y_{N-1} Y\left(\left|y_{N-1}-y\right|, Q_{N-1}, Q\right) \\
& \cdots \\
& \int_{2}^{Q_{2}} d Q_{1} p_{a}\left(Q_{1} \mid Q_{2}\right) \int_{-\infty}^{\infty} d y_{1} Y\left(\left|y_{1}-y_{2}\right|, Q_{1}, Q_{2}\right) \\
& p_{a}\left(Q_{f} \mid Q_{1}\right) Y\left(\left|y_{f}-y_{1}\right|, Q_{f}, Q_{1}\right),
\end{aligned}
$$


by using the same technique discussed in the previous Subsection (Gaussian approximation of $\left.Y\left(\left|y_{i}-y_{i+1}\right|, Q_{i}, Q_{i+1}\right)\right)$, one finds:

$$
r_{N}\left(Q_{f} y_{f} \mid Q y\right)=p_{a}\left(Q_{f} \mid Q\right) Y\left(\left|y_{f}-y\right|, Q_{f}, Q\right) \frac{\lambda(Q)^{N-1}}{(N-1) !} .
$$

The structure of this calculation is very similar to that performed in [4] (Eq. (48)). The main difference lies in the fact that here we are studying the production mechanism for partons inside a clan and not for clans themselves, i.e., the result must depend on the new parameter $a$ only.

Finally, one should notice that in this scheme the ancestor parton after the splitting continues on its way whereas the daughter parton gives origin to the clan. Therefore partons' MD inside the clan is shifted by one unit.

Our calculation can be simplified by assuming $Q_{f}=1 \mathrm{GeV}$ in $Y\left(\mid y_{f}-\right.$ $\left.y \mid, Q_{f}, Q\right)$, an assumption which is verified for clans of high virtuality and is quite acceptable in general. Accordingly, one has:

$$
\rho\left(y_{f} \mid Q y\right)=e^{\lambda_{a}(Q)} \int_{1}^{2} d Q_{f} p_{a}\left(Q_{f} \mid Q\right) Y\left(\left|y_{f}-y\right|, Q_{f}, Q\right)
$$

which, by using Eqs. (35) and (41), becomes

$$
\rho\left(y_{f} \mid Q y\right)=\frac{1}{2 \log Q} \theta\left(\log Q-\left|y_{f}-y\right|\right) .
$$

Equation (71) is valid for $Q \geq 2 \mathrm{GeV}$. For $Q<2 \mathrm{GeV}$, one produces clans with only one parton, i.e.:

$$
\rho_{Q<2}\left(y_{f} \mid Q y\right)=\delta\left(y-y_{f}\right) .
$$

Having found $\rho_{Q<2}\left(y_{f} \mid Q y\right)$, we can now calculate from Eqs. (52) and (49) the average number of partons in a single clan:

$$
\begin{gathered}
\bar{n}_{c}(\Delta y, Q, y)=e^{\lambda_{a}(Q)} \max \left[0, \frac{\min (\sup (\Delta y), y+\log Q)-\max (\inf (\Delta y), y-\log Q)}{2 \log Q}\right] \\
\text { for } \quad Q \geq 2 \mathrm{GeV}
\end{gathered}
$$

and

$$
\begin{aligned}
\bar{n}_{c}(\Delta y, Q, y) & =e^{\lambda_{a}(Q)} \quad \begin{array}{l}
y \in \Delta y \\
\text { for } Q<2 \mathrm{GeV}
\end{array}
\end{aligned}
$$

respectively. 


\subsection{The average number of partons in $\Delta y$ in an average clan}

The main content of this Section concerns the explicit analytical expression of $\bar{n}_{c}(\Delta y, W)$. This result follows by solving the double integral over $Q$ and $y$ in Eq. (51); $\bar{n}_{c}(\Delta y, Q, y)$ is given here by Eqs. (73) and (74), whereas $\sigma(Q, y \mid W)$ is obtained by inserting Eqs. (63) and (64) into Eq. (53).

Attention should be paid to the integration domain of $y$ and $Q$, which is determined by the conditions $1 \mathrm{GeV} \leq Q \leq W$ and $-\log W / 2 Q \leq y \leq$ $\log 2 W / Q$ (see Eq. (65))). Notice that according to Eqs. (73) and (74) the integrand has a different structure in the intervals $1 \mathrm{GeV} \leq Q \leq 2 \mathrm{GeV}$ and 2 $\mathrm{GeV} \leq Q \leq W \mathrm{GeV}$. Accordingly, we perform separately the two integrations:

$$
\bar{n}_{c}(\Delta y, W)=\bar{n}_{c}(Q<2)+\bar{n}_{c}(Q \geq 2) .
$$

In the interval $1 \mathrm{GeV} \leq Q \leq 2 \mathrm{GeV}$, one has:

$$
\begin{aligned}
\bar{n}_{c}(Q<2)= & \int_{1}^{2} d Q \int_{-\log W / 2 Q}^{\log 2 W / Q} d y \theta\left(y_{c}-|y|\right) p_{A}(Q \mid W) C_{A}(2) \\
& \int_{\max [\log 2,(\log 2 W-y+\log Q) / 2]}^{\log W} d \log Q^{\prime} Q^{\prime} \frac{p_{0}^{A}\left(Q^{\prime}\right)}{2 \log Q^{\prime} / Q}+ \\
+\int_{1}^{2} d Q \quad & \int_{-\log W / 2 Q}^{\log 2 W / Q} d y \theta\left(y_{c}-|y|\right) p_{A}(Q \mid W) \delta\left(y-\tanh ^{-1} \sqrt{1-(Q / W)^{2}}\right) .
\end{aligned}
$$

In the approximation of Eq. (62), Eq. (76) can be rewritten as follows:

$$
\begin{aligned}
\bar{n}_{c}(Q<2) & =\int_{1}^{2} d Q p_{A}(Q \mid W) C_{A}(2) \Sigma^{A}(Q, y, W)+ \\
& +\theta\left(y_{c}-\log W\right)\left[\left(\frac{\log 2}{\log W}\right)^{A}-\left(\frac{\log 2 W-y_{c}}{\log W}\right)^{2}\right],
\end{aligned}
$$

where

$$
\begin{aligned}
& \Sigma^{A}(Q, y, W) \equiv \int_{(\log 2 W-y+\log Q) / 2}^{\log W} d \log Q^{\prime} Q^{\prime} \frac{p_{0}^{A}\left(Q^{\prime}\right)}{2 \log Q^{\prime} / Q}= \\
& \frac{A}{2}(\log Q)^{A-1}\left\{\log x+\sum_{n=1}^{\infty}\left(\begin{array}{c}
A-1 \\
n
\end{array}\right) \frac{x^{n}}{n}\right\}_{(\log W-y+\log Q) / 2 \log Q-1}^{\log W / \log Q-1}
\end{aligned}
$$

In particular, for $A=2$, one gets:

$$
\begin{aligned}
\Sigma^{2}(Q, y, W) & =\log Q\left[\log \left(\frac{\log W}{\log Q}-1\right)-\log \left(\frac{\log 2 W-y+\log Q}{2 \log Q}-1\right)\right]+ \\
& +\frac{y}{2}+\frac{1}{2} \log W / 2 Q .
\end{aligned}
$$


Coming to the interval $2 \mathrm{GeV} \leq Q \leq W$, one can split the integration in two different terms:

$$
\bar{n}_{c}(Q \geq 2)=\bar{n}_{c}(Q \geq 2 ; \alpha)+\bar{n}_{c}(Q \geq 2 ; \beta),
$$

where

$$
\begin{aligned}
& \bar{n}_{c}(Q \geq 2 ; \alpha)=\int_{2}^{W} d Q \int_{-\log W / 2 Q}^{\log 2 W / Q} d y \frac{e^{\lambda_{a}(Q)}}{\bar{N}(\mathrm{fps}, W)} \\
& \max \left[0, \frac{\min (\sup (\Delta y), y+\log Q)-\max (\inf (\Delta y), y-\log Q)}{2 \log Q}\right] \\
& p_{A}(Q \mid W) \delta\left(y-\tanh ^{-1} \sqrt{1-(Q / W)^{2}}\right)
\end{aligned}
$$

and

$$
\begin{aligned}
& \bar{n}_{c}(Q \geq 2 ; \beta)=\int_{2}^{W} d Q \int_{-\log W / 2 Q}^{\log 2 W / Q} d y \frac{e^{\lambda_{a}(Q)}}{\bar{N}(\mathrm{fps}, W)} \\
& \max \left[0, \frac{\min (\sup (\Delta y), y+\log Q)-\max (\inf (\Delta y), y-\log Q)}{2 \log Q}\right] \\
& p_{A}(Q \mid W) C_{A}(Q) \Sigma^{A}(Q, y, W) .
\end{aligned}
$$

So far the rapidity interval has been generic, but in order to continue our explicit calculations in agreement with standard practice 1 , we specialize in the following to central symmetric intervals of rapidity of half-width $y_{c}: \Delta y=$ $\left[-y_{c}, y_{c}\right]$.

By using Eq. (62) and the properties of the $\delta$-function, one has:

$$
\begin{aligned}
\bar{n}_{c}(Q \geq 2 ; \alpha)= & \int_{2}^{W} d Q \frac{1}{2 \log Q} \frac{C_{a}(2) p_{0}^{A}(Q) C_{A}(W)}{C_{a}(Q) \bar{N}(\mathrm{fps}, W)} \\
& \max \left[0, \min \left(y_{c}, \log 2 W\right)-\max \left(-y_{c}, \log 2 W-2 \log Q\right)\right] .
\end{aligned}
$$

Notice that the expression of the maximum in Eq. (83) assumes the following different values in different ranges of $\log Q$, i.e., 0 for $\log Q<\frac{1}{2}\left(\log 2 W-y_{c}\right)$, $y_{c}-\log 2 W+2 \log Q$ for $\frac{1}{2}\left(\log 2 W-y_{c}\right) \leq \log Q \leq \frac{1}{2}\left(\log 2 W+y_{c}\right)$ and $2 y_{c}$ for $\frac{1}{2}\left(\log 2 W+y_{c}\right)<\log Q<\log 2 W$. It follows:

$$
\begin{gathered}
\bar{n}_{c}(Q \geq 2 ; \alpha)=\frac{1}{(\log 2)^{a}(\log W)^{A} \bar{N}(W)}\left\{\left(y_{c}-\log 2 W\right) \frac{A}{2(A+a-1)}\right. \\
{\left[\left(\frac{\log 2 W+y_{c}}{2}\right)^{A+a-1}-\left(\frac{\log 2 W-y_{c}}{2}\right)^{A+a-1}\right]+} \\
+\frac{A}{A+a}\left[\left(\frac{\log 2 W+y_{c}}{2}\right)^{A+a}-\left(\frac{\log 2 W-y_{c}}{2}\right)^{A+a}\right]+
\end{gathered}
$$




$$
\left.+y_{c} \frac{A}{A+a-1}\left[(\log W)^{A+a-1}-\left(\frac{\log 2 W+y_{c}}{2}\right)^{A+a-1}\right]\right\}
$$

for $y_{c}<\log W / 2$ and

$$
\begin{aligned}
& \bar{n}_{c}(Q \geq 2 ; \alpha)=\frac{1}{(\log 2)^{a}(\log W)^{A} \bar{N}(W)}\left\{\left(y_{c}-\log 2 W\right) \frac{A}{2(A+a-1)}\right. \\
& {\left[(\log W)^{A+a-1}-(\log 2)^{A+a-1}\right]+} \\
+ & \left.\frac{A}{A+a}\left[(\log W)^{A+a}-(\log 2)^{A+a}\right]\right\}
\end{aligned}
$$

for $y_{c} \geq \log W / 2$.

Now, we calculate the second term, $\bar{n}_{c}(Q \geq 2 ; \beta)$. It is convenient to introduce a new set of variables, i.e.:

$$
\begin{aligned}
& \xi=\log Q+y \\
& \eta=\log Q-y .
\end{aligned}
$$

In these variables the initial integration domain turns out to be:

$$
\eta \leq \log \left(\frac{W}{2}\right), \quad \xi \leq \log (2 W), \quad \eta+\xi>2 \log 2,
$$

as indicated in Figure (1) (solid line).

Notice also that the expression of the maximum contained in Eq. (73) assumes different values in different sectors of the integration domain.

For $y_{c}$ in the interval $(\log 2 \div \log W / 2)$ one can identify four sectors (see Figure (1), dotted lines):

I)

$$
y_{c}<\xi<\log 2 \quad y_{c}<\eta<\log W / 2
$$

$I I)$

$$
\begin{array}{lr}
-y_{c}+2 \log 2<\xi<y_{c} & y_{c}<\eta<\log W / 2 \\
\max \left(-y_{c},-\log W / 8\right)<\xi<-y_{c}+2 \log 2 & -\xi+2 \log 2<\eta<\log W / 2 ;
\end{array}
$$

$I I I)$

$$
\begin{aligned}
& y_{c}<\xi<\log 2 W \quad-y_{c}+2 \log 2<\eta<y_{c} \\
& -\eta+2 \log 2<\xi<\log 2 W \quad \max \left(-y_{c},-\log W / 2\right)<\eta<-y_{c}+2 \log 2 ;
\end{aligned}
$$

$I V)$

$$
-y_{c}+2 \log 2<\xi<y_{c} \quad-\xi+2 \log 2<\eta<y_{c}
$$


where $\pi_{a}(\Delta y, Q, y)$ is:

$$
\begin{array}{cl}
I) & 2 y_{c} \\
I I) & \xi+y_{c} \\
I I I) & \eta+y_{c} \\
I V) & \xi+\eta
\end{array}
$$

respectively.

For $y_{c}$ values in the extreme allowed interval, $y_{c} \geq \log W / 2$, sectors $I$ ) and $I I)$ disappear and the other two sectors $I I I$ and $I V$ are modified as follows: $I I I)$

$$
y_{c}<\xi<\log 2 W \quad-\xi+2 \log 2<\eta<\log W / 2 ;
$$

$I V)$

$$
-\log W / 8<\xi<y_{c} \quad-\xi+2 \log 2<\eta<\log W / 2 .
$$

Finally, for $y_{c} \leq \log 2$, sector $I V$ ) only disappears and the others sectors become:

I)

$$
\begin{array}{cc}
y_{c}<\xi<\log 2 W \quad-y_{c}+2 \log 2<\eta<\log W / 2 \\
-\eta+2 \log 2<\xi<\log 2 W \quad y_{c}<\eta<-y_{c}+2 \log 2 ;
\end{array}
$$

II)

$$
-y_{c}<\xi<y_{c} \quad-\xi+2 \log 2<\eta<\log W / 2 ;
$$

$I I I)$

$$
-\eta+2 \log 2<\xi<\log 2 W \quad-y_{c}<\eta<y_{c} .
$$

The final analytical result for $\bar{n}_{c}(Q \geq 2 ; \beta)$ can then be obtained. In order to have an impression of the structure of this formula, we show it explicitly in the domain $\log 2 \leq y_{c} \leq \log W$ :

$$
\begin{gathered}
\bar{n}_{c}(Q \geq 2, \beta)=\frac{1}{1+A \log \left(\frac{(\log W)}{(\log 2)}\right)}\left\{4 y_{c}+\frac{7 y_{c}}{2(\log W)}+\frac{17 y_{c}}{12(\log 2)}+\right. \\
+\frac{29(\log 2) y_{c}}{12(\log W)^{2}}-\frac{2(\log 2)^{2} y_{c}}{3(\log W)^{2}}+\frac{7 y_{c}{ }^{3}}{12(\log W)^{2}(\log 2)}+
\end{gathered}
$$




$$
\begin{aligned}
& +\frac{4(\log W) y_{c} \log ((\log W))}{3(\log 2)^{2}}+\frac{2 y_{c} \log ((\log W))}{(\log 2)}+ \\
& +\frac{2\left(-2(\log W)^{3} y_{c}+3(\log W)^{2}(\log 2) y_{c}-(\log 2)^{3} y_{c}\right) \log ((\log W)-(\log 2))}{3(\log W)^{2}(\log 2)^{2}}+ \\
& +\frac{\left(-(\log W)+y_{c}\right)^{2} \log \left((\log W)-y_{c}\right)}{6(\log W)^{2}(\log 2)^{2}}\left[-(\log W)^{2}-4(\log W)(\log 2)+\right. \\
& \left.-6(\log 2)^{2}+2(\log W) y_{c}+4(\log 2) y_{c}-y_{c}^{2}\right]+ \\
& +\frac{\left((\log W)-(\log 2)-y_{c}\right)^{3} \log \left((\log W)-(\log 2)-y_{c}\right)}{12(\log W)^{2}(\log 2)}+ \\
& +\frac{\left(-(\log W)-(\log 2)+y_{c}\right)^{2} \log \left((\log W)+(\log 2)-y_{c}\right)}{12(\log W)^{2}(\log 2)^{2}}\left[2(\log W)^{2}+\right. \\
& \left.+15(\log W)(\log 2)-5(\log 2)^{2}-4(\log W) y_{c}-3(\log 2) y_{c}+2 y_{c}{ }^{2}\right]+ \\
& +\frac{\left((\log W)+y_{c}\right)^{2} \log \left((\log W)+y_{c}\right)}{6(\log W)^{2}(\log 2)^{2}}\left[(\log W)^{2}+4(\log W)(\log 2)+\right. \\
& \left.+6(\log 2)^{2}+2(\log W) y_{c}+4(\log 2) y_{c}+y_{c}^{2}\right]+ \\
& +\frac{\left(-(\log W)+(\log 2)-y_{c}\right)^{3} \log \left((\log W)-(\log 2)+y_{c}\right)}{12(\log W)^{2}(\log 2)}+ \\
& +\frac{\left((\log W)+(\log 2)+y_{c}\right)^{2} \log \left((\log W)+(\log 2)+y_{c}\right)}{12(\log W)^{2}(\log 2)^{2}}\left[-2(\log W)^{2}+\right. \\
& \left.\left.-15(\log W)(\log 2)+5(\log 2)^{2}-4(\log W) y_{c}-3(\log 2) y_{c}-2 y_{c}^{2}\right]\right\} \text {. }
\end{aligned}
$$

Having now integrated, as proposed in Eq. (51), the product of the average parton multiplicity in a rapidity interval $\Delta y$ for a single clan of given virtuality $Q$ and rapidity $y$, times the probability that a parton of maximum allowed virtuality $W$ produces a clan of virtuality $Q$ and rapidity $y$, by dividing the integration domain in several regions, one obtains the wanted final analytical expression for the average number of partons inside an average clan in the interval $\Delta y$ by assembling the various pieces as follows:

$$
\begin{aligned}
\bar{n}_{c}(\Delta y, W) & =\bar{n}_{c}(Q<2)+\bar{n}_{c}(Q \geq 2) \\
& =\bar{n}_{c}(Q<2)+\bar{n}_{c}(Q \geq 2 ; \alpha)+\bar{n}_{c}(Q \geq 2 ; \beta) .
\end{aligned}
$$

The average number of partons in the shower, $\bar{n}(\Delta y, W)$, can then be calculated from Eq. (31). 


\section{Results: comments and discussion}

The motivation of this work - as pointed out in the Introduction - has been to justify from first principle clan structure analysis in multiparticle production and to provide a sound theoretical basis to the regularities discovered by its application to experimental data. In order to do that, one would need QCD calculations of a bi-dimensional shower evolution in a region where QCD predictions are not available and calculations are very hard to be done. One should therefore fully rely on models. Our program was to study analytically clan structure in rapidity and virtuality variables in a parton shower model based on essentials of QCD in a correct kinematical framework, the GSPS model. Results of analytical calculations of the average number of clans behavior in virtuality and rapidity variables were reported in [4] and summarized in the introduction. In order to complete our initial program we propose in this paper to discuss the properties of the second parameter of clan structure analysis, i.e., of the average number of partons per clan, in rapidity and virtuality variables as resulting from the analytical calculations presented in previous Sections.

Notice that these calculations have been performed by following very mild mathematical assumptions and by choosing for the parameters $A$ and $a$ of the GSPS model the values 2 and 1 respectively. It should be remembered that $A$ and $a$ control the "length" of the parton shower and of clans in the shower. The suggested choices avoid nasty inessential calculations and make possible the analytical solution of the model without hurting its logic. It should be pointed out that MC calculations based on the same architecture of the GSPS model allow to predict the behavior of $\bar{n}_{c}(\Delta y, W)$ as well as that of the average number of clans in the same variables, $\bar{N}(\Delta y, W)$, for all values of the parameters $a$ and $A$. In addition MC calculations here can be considered as exact, i.e., the result is obtained without any of the above mentioned mild mathematical approximations used in the analytical calculation in order to get the explicit dependence of $\bar{N}(\Delta y, W)$ and $\bar{n}_{c}(\Delta y, W)$ on $\Delta y$ and $W$. This fact should be considered important for estimating the goodness of the assumptions performed in the analytical calculations.

Knowing from previous work 4 the rapidity and virtuality dependence of the average number of clan, the analytical calculation of the average number of partons per clan in the same variables can be done in terms of the average number of partons in the full shower. This is indeed a quantity which as previously discussed - does not depend on the redefinition procedure and therefore can safely be used to our purpose.

The result of the analytical calculation of the average number of partons in the shower, $\bar{n}(\Delta y, W)$, as a function of the rapidity interval $\Delta y$ and of 
the maximum allowed virtuality $W$ with $A=2$ and $a=1$, Eq. (29), is shown in Figure (2) for $W=50 \mathrm{GeV}$ (solid line), $W=100 \mathrm{GeV}$ (dashed line) and $W=$ $500 \mathrm{GeV}$ (dotted line). The average parton multiplicity grows almost linearly with rapidity for relatively small $\Delta y$ intervals and then it is slowly bending for $\Delta y$ intervals approaching to fps, where it reaches its maximum $(\bar{n}(\mathrm{fps}) \simeq 10$ for $W=50 \mathrm{GeV}, \bar{n}$ (fps) $\simeq 12$ for $W=100 \mathrm{GeV}$ and $\bar{n}(\mathrm{fps}) \simeq 17$ for $W=500$ $\mathrm{GeV})$.

Monte Carlo calculations of $\bar{n}\left(y_{c}, W\right)$ for the same $W$ values and for the same choice of the parameters $A$ and $a$ are given in Figure (3). The general trend is the same as that in Figure (2). The agreement between the two calculations is very good in fps. For smaller $\Delta y$ intervals Monte Carlo calculations are sistematically higher into few percent and allow to estimate the amount of the approximations done in the analytical calculation.

It is interesting to remark that the normalized average number of partons in the shower, $\bar{n}(\Delta y, W) / \bar{n}(\mathrm{fps}, W)$ scales in virtuality as a function of the rescaled rapidity interval, $\Delta y / \mathrm{fps}$, see Figure (国). This scaling in $W$ is found to depend on the parameter $a$, as different values of $a$ give different scaling curves, differently from the scaling found in [4] for $\bar{N}(\Delta y, W) / \bar{N}(\mathrm{fps}, W)$ which is independent of the mechanism inside clans.

Figure (5a) shows the behavior of the average number of clans, $\bar{N}^{\prime}(\Delta y, W)$, in showers of maximum allowed virtuality $W$ equal to $50 \mathrm{GeV}$ (solid line), 100 $\mathrm{GeV}$ (dashed line) and $500 \mathrm{GeV}$ (dotted line) as a function of rapidity interval $\Delta y$ (Eq. (28)). The analytical calculation has been done with $A=2$. Being the first step only of the production process involved in the calculation, the result does not depend of course on the parameter $a$. The Figure content is the same discussed in Figure 8 of 4 ].

Having shown in Figures (2) and (5a) the general trends of the average number of partons in the shower and of the average number of clans as functions of rapidity and virtuality variables predicted by the analytical calculations according to Section 4 and our previous work 4 , we are now ready to calculate the average number of partons per clan in the same variables, i.e., $\bar{n}_{c}(\Delta y, W)$, simply by forming the ratio of the above two quantities.

$\bar{n}_{c}(\Delta y, W)$ values in rapidity intervals for $W=50 \mathrm{GeV}$ (solid line), $W=$ $100 \mathrm{GeV}$ (dashed line) and $W=500 \mathrm{GeV}$ (dotted line), with parameters $A$ and $a$ equal to 2 and 1 respectively, are plotted in Figure (5) b). The result is reasonable although not fully satisfactory. An anomalous behavior is clearly visible for large rapidity intervals $\left(\bar{n}_{c}(\Delta y, W)\right.$ increases as $\Delta y \rightarrow \mathrm{fps}$ in contrast with what one would expect from standard clan structure analysis at parton level) and for small rapidity intervals $\left(\bar{n}_{c}(\Delta y, W)\right.$ is shown to point to constant values, which differ for different $W$ values in the limit $\Delta y \rightarrow 0$ in contrast with the expected $W$ independent $\bar{n}_{c}(0, W)=1$ value). In order to cure the first 
anomaly we decided to use the definition from the CPD form, Eq. (21), which is relevant in fps, and accordingly to use Eq. (23) for the average number of partons per clan. The relevance of this change is shown in Figure (6a), where $\lambda^{\prime}(\Delta y, W)$ is shown to diverge as $\Delta y \rightarrow$ fps as expected; because of this behavior, however, the procedure cannot be extended to fps. It will be noticed in Figure (6) $\mathrm{b}$ ) that indeed the above mentioned anomaly is removed: the result becomes consistent with our scheme by assuming that only clans with at least one parton are allowed.

The most natural motivation of the appearence of the second anomaly lies in our opinion in the approximations used in the analytical calculations as already shown by comparing Figures (2) and (3). The approximations imply deviations from the exact behavior for $\Delta y<$ fps. In order to check this guess we propose to calculate the average number of clans and the average number of partons per clan with the MC. Results of the MC calculations are shown in Figures $(7 \mathrm{7})$ and (7) $\mathrm{b})$. Their trends fully coincide with standard results already observed in clan structure analysis applied to parton showers in quark and gluon jets. The predictive power of the model under investigation is therefore confirmed. In addition, for small rapidity intervals the expected $\bar{n}_{c}(\Delta y=0, W)=1$ limit is recovered.

We have shown that the parton shower model, which we built by assuming QCD-inspired dependence of the splitting functions in virtuality and in rapidity and Sudakov form factors for their normalization ("essentials of QCD"), and by allowing at each step in the cascading local violations of energy-momentum conservation law, but requiring its global validity, has an extraordinary predictive power in regions not accessible to full perturbative QCD. In particular, we performed analytical calculations of the rapidity and virtuality dependence of the average number of clans, of the average parton multiplicity and of the average number of partons inside a clan in a parton shower initiated by an ancestor parton of given virtuality. Clans here should be intended of course in a more general sense than that usually referred to in the literature and linked hystorically to NB regularity: they are the natural language for describing a two step process in terms of a CPD and can be identified in our parton shower model with effective intermediate independent parton (gluon) sources in the shower. Results of our analytical calculations are consistent with what we know on clan properties in single quark and gluon jets disentangled by using a jet finding algorithm and analyzed at parton level by assuming the validity of GLPHD. In addition it should be pointed out that, even if the change of $A$ and $a$ parameters do not influence the qualitative behavior of the average number of clans and the average number of partons per clan in rapidity and virtuality, it actually affects their numerical values. Since $A$ and $a$ parameters control the length of the parton shower and the development of parton cascad- 
ing inside a clan, would one describe a two parton species shower initiated by an ancestor gluon or quark, this flexibility of the model could be very useful in order to describe jets of different origin. This mechanism could be also very helpful in a more general framework for explaining the observed different behavior of clans in different classes of high energy collisions. In summary, these findings suggest that the dynamics of multiparticle production is controlled by a QCD inspired two step process dominated by independent intermediate parton (gluon) sources, which we call clans, and by their cascading. The features which make the model appealing are its simple structure, the possibility to calculate in its framework analytically important physical quantities and its flexibility.

\section{Acknowledgement}

One of us (S.L.) would like to thank D.A.A.D. for financial support.

\section{References}

[1] N. Schmitz, in Multiparticle Dynamics (Festschrift for Léon Van Hove), La Thuile, Italy, eds. A. Giovannini and W. Kittel, World Scientific, Singapore, 1990, p. 25

[2] L. Van Hove and A. Giovannini, Acta Phys. Pol. B19 (1988) 917

[3] W. Ochs, in Proc. XXIV International Symposium on Multiparticle Dynamics (Vietri sul mare (SA), Italy, 1994), eds. A. Giovannini, S. Lupia, R.Ugoccioni, World Scientific, Singapore, 1995, p. 243

[4] R. Ugoccioni, A. Giovannini and S. Lupia, Z. Phys. C - Particles and Fields 64 (1994) 453

[5] F. Bianchi, A. Giovannini, S. Lupia, R. Ugoccioni, Z. Phys. C - Particles and Fields 58 (1993) 71.

[6] S. Lupia, A. Giovannini, R. Ugoccioni, Z. Phys. C-Particles and Fields 66 (1995) 195

[7] S. Lupia, A. Giovannini, R. Ugoccioni, Z. Phys. C - Particles and Fields 59 (1993) 427

[8] A. Giovannini and L. Van Hove, Z. Phys. C - Particles and Fields 30 (1986) 391. 
[9] S. Hegyi, Phys.. Lett. B318 (1993) 642

[10] R. Ugoccioni and A. Giovannini, Z. Phys. C - Particles and Fields 53 (1992) 239 .

[11] V. N. Gribov, L. N. Lipatov, Sov. J. Nucl. Phys. 15 (1972) 438, 675; G. Altarelli and G. Parisi, Nucl. Phys. B126 (1977) 298

[12] K. Kajantie and E. Pietarinen, Phys. Lett. B93 (1980) 269

[13] B. Andersson, Nucl. Phys. B360 (1991) 109; G. Gustafson, Nucl. Phys. B392 (1993) 251; G. Gustafson and M. Olsson, Nucl. Phys. B406 (1993) 293.

[14] A. Giovannini and L. Van Hove, Acta Phys. Pol. B19 (1988) 495

[15] A. Giovannini, Nucl. Phys. B161 (1979) 427 


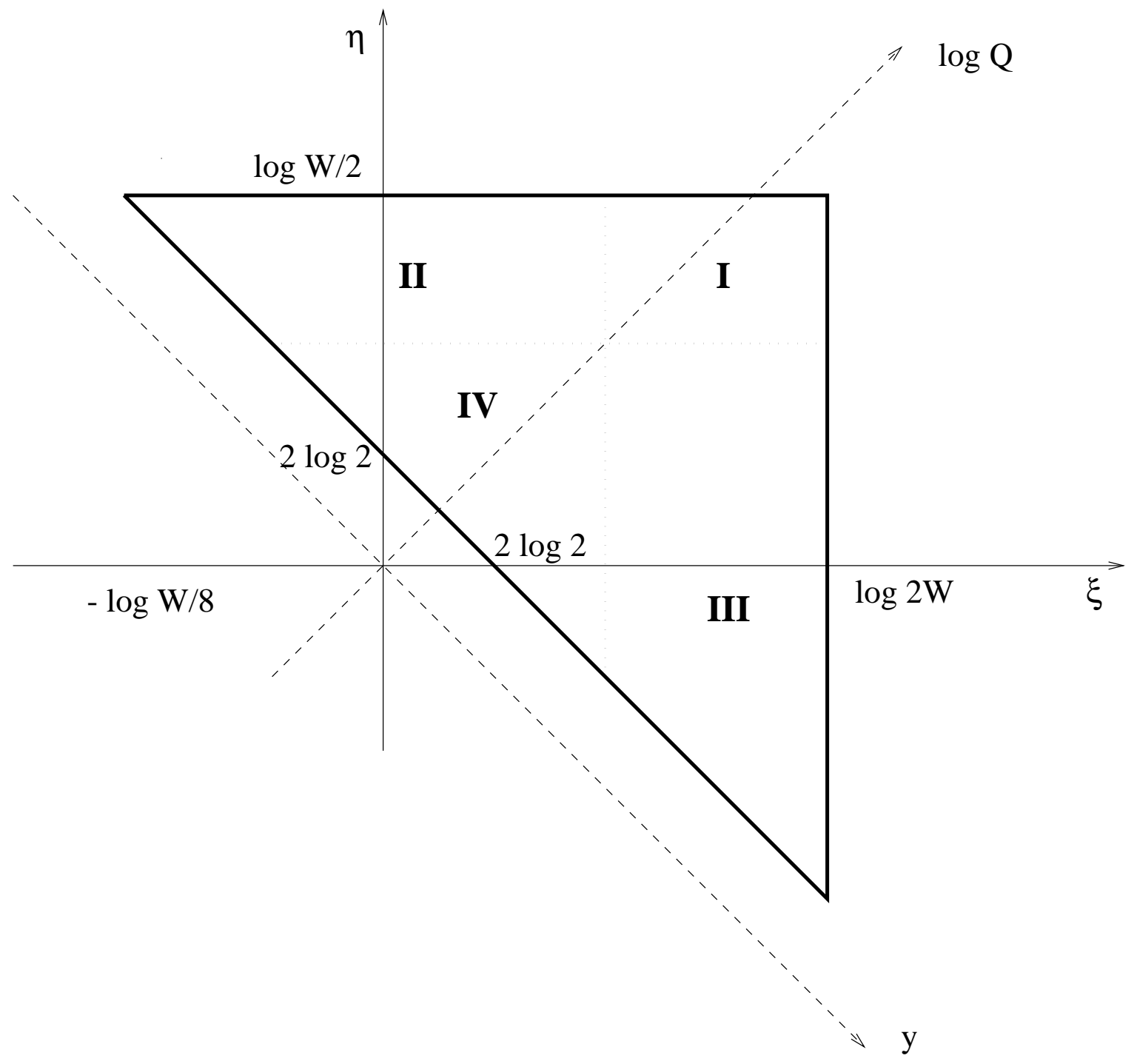

Figure 1: Integration domain in the $(\xi, \eta)$ plane for $\bar{n}_{c}(Q \geq 2, \alpha)$ (see text, Eq. (88)). Dotted lines indicate phase space domains where the integrand assumes different values. 


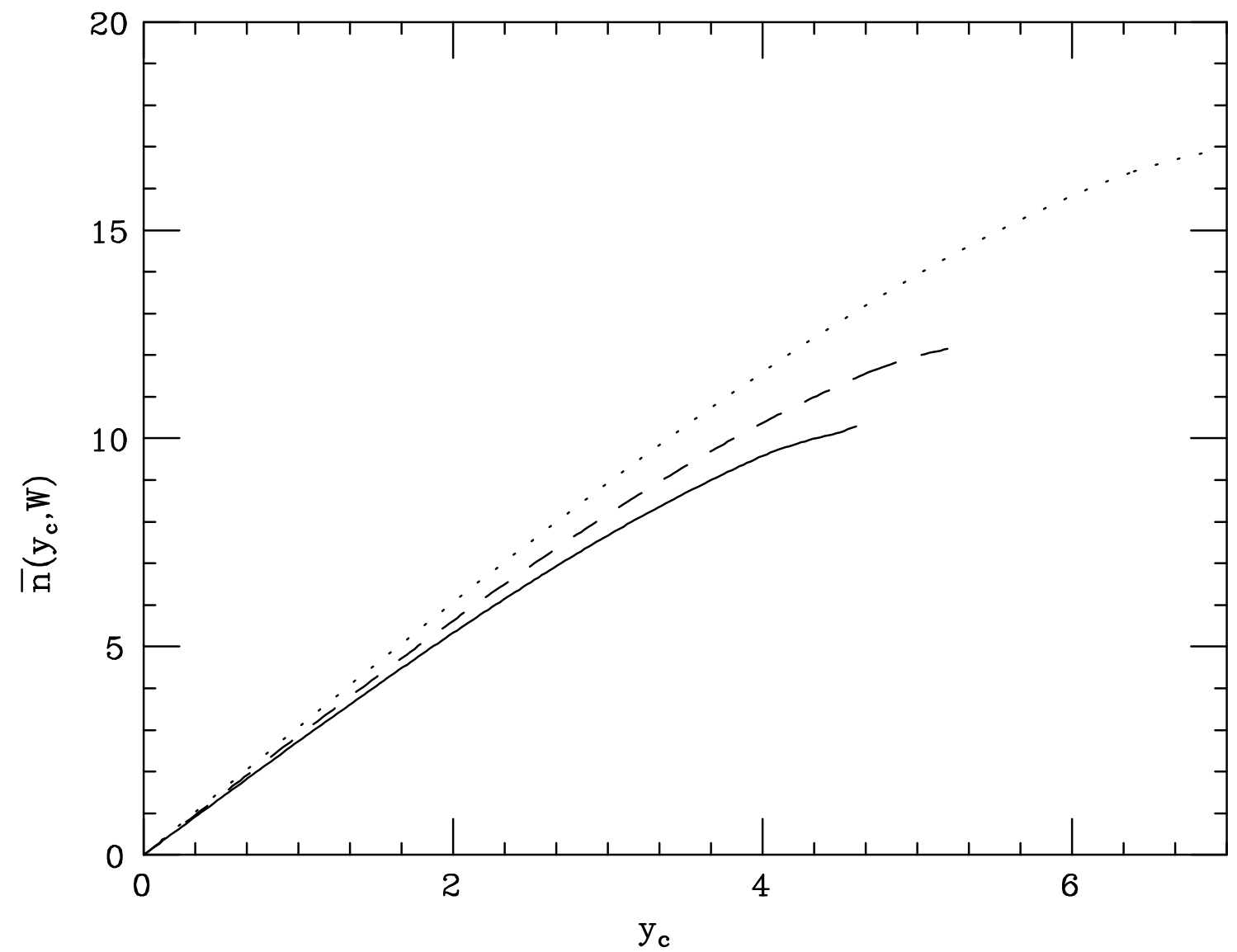

Figure 2: Average number of partons in the shower, $\bar{n}\left(y_{c}, W\right)$, as a function of the width of the rapidity interval $y_{c}$ obtained analytically in the GSPS model with $A=2, a=1$ at different maximum allowed virtualities $W=50 \mathrm{GeV}$ (solid line), $100 \mathrm{GeV}$ (dashed line) and $500 \mathrm{GeV}$ (dotted line) (see Eq. (29)). 


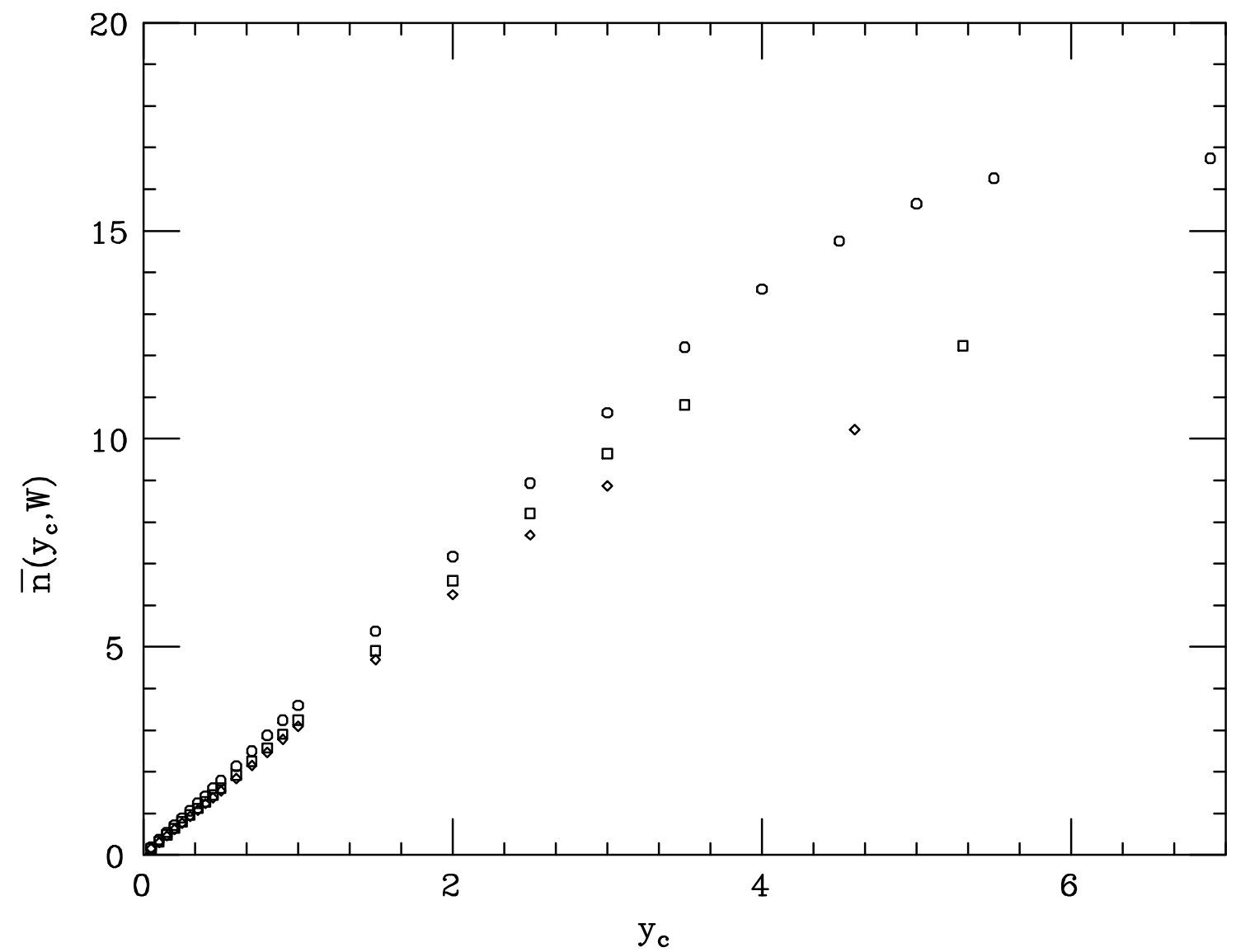

Figure 3: Same as in Figure (2), but for the Monte Carlo version of the model at different maximum allowed virtualities $W=50 \mathrm{GeV}$ (diamonds), $100 \mathrm{GeV}$ (squares) and $500 \mathrm{GeV}$ (circles). 


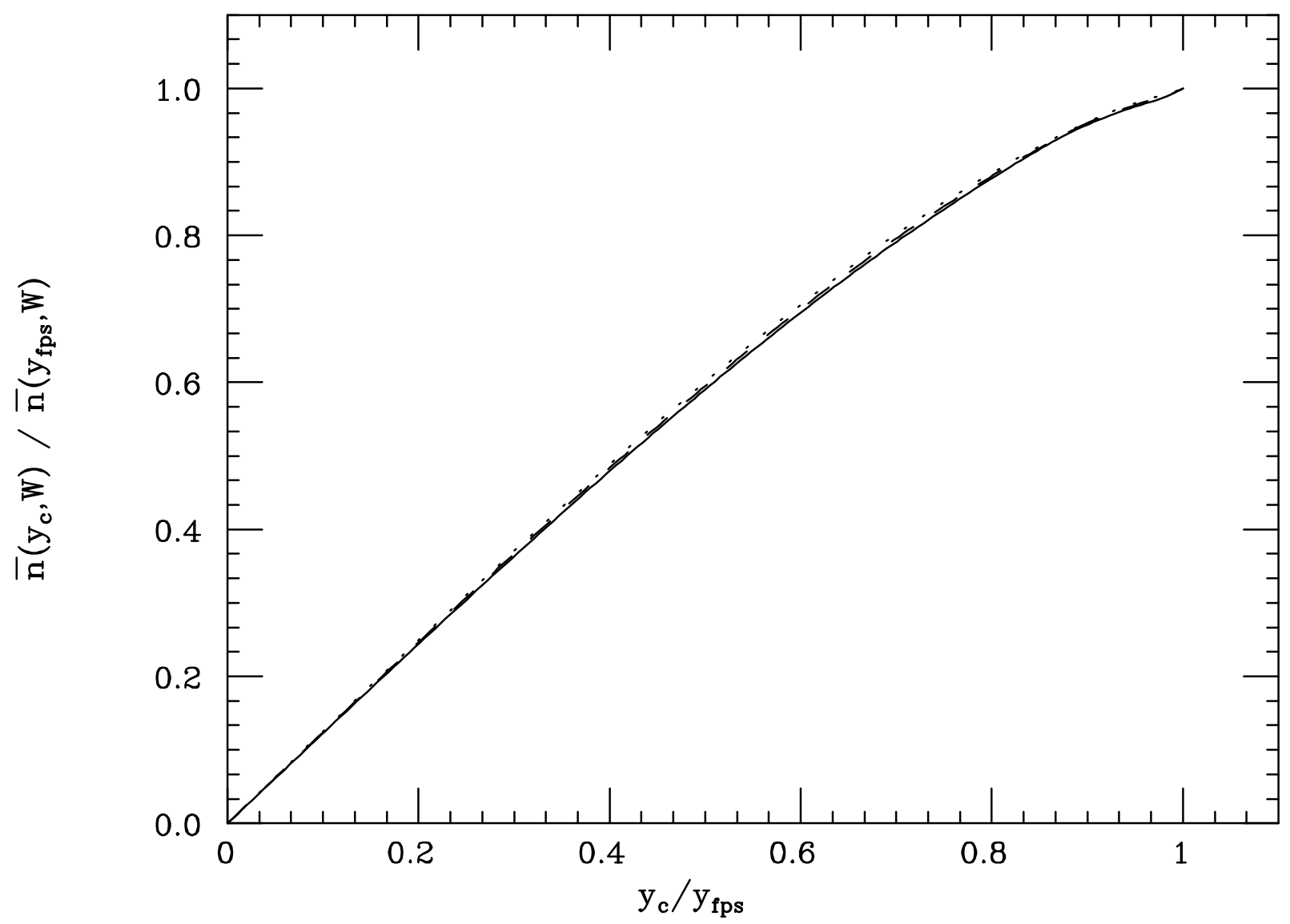

Figure 4: Normalized average number of partons in the shower, $\bar{n}\left(y_{c}, W\right) / \bar{n}(\mathrm{fps}, W)$ as a function of rescaled rapidity interval, $y_{c} /$ fps for $A$ $=2, a=1$ at different maximum allowed virtualities $W=50 \mathrm{GeV}$ (solid line), $100 \mathrm{GeV}$ (dashed line) and $500 \mathrm{GeV}$ (dotted line). 

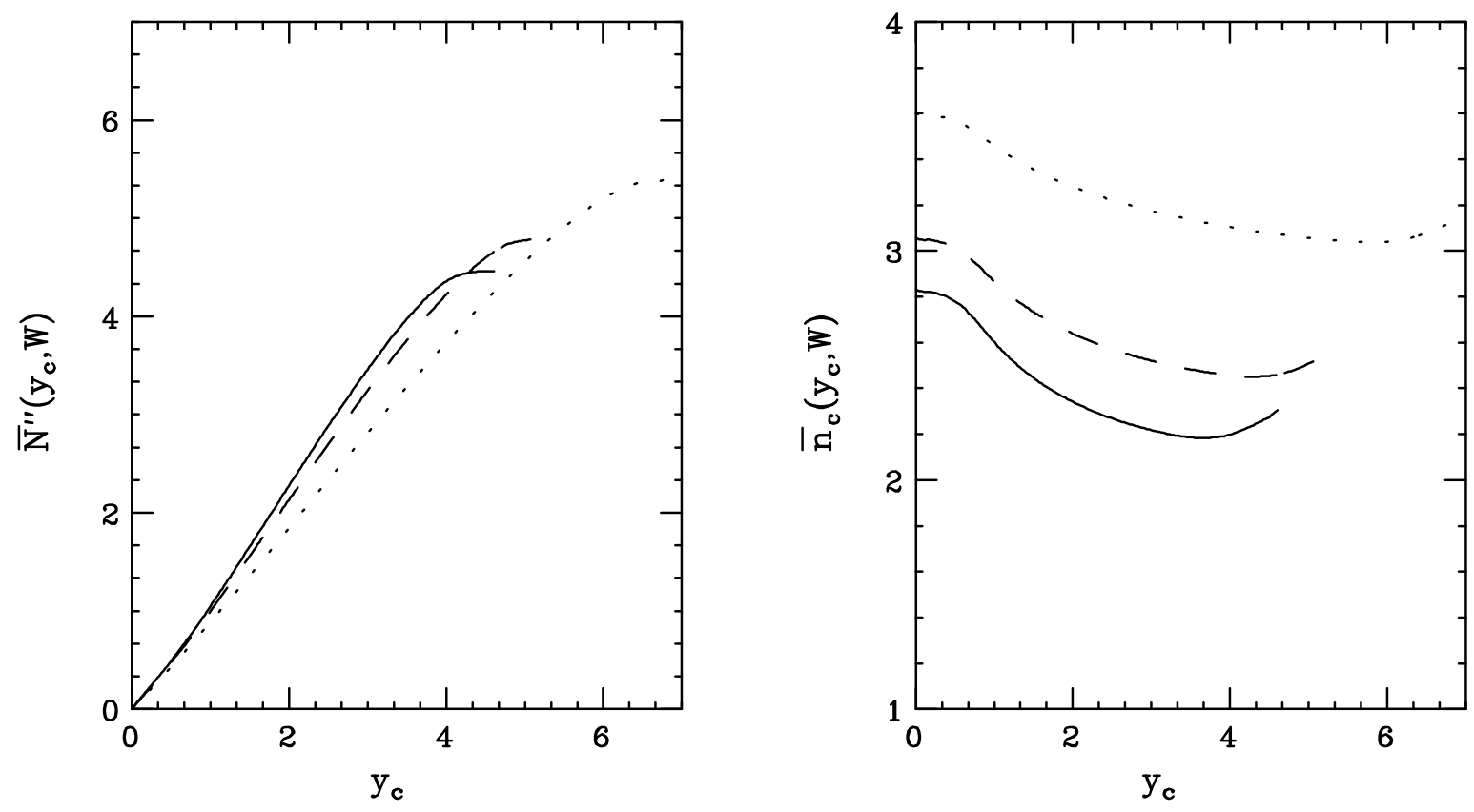

Figure 5: a): average number of clans, $\bar{N}^{\prime \prime}\left(y_{c}, W\right)$, defined by binomial convolution as in Eq. (28), as a function of the width of the rapidity interval $y_{c}$ at different maximum allowed virtualities $W=50 \mathrm{GeV}$ (solid line), $100 \mathrm{GeV}$ (dashed line) and $500 \mathrm{GeV}$ (dotted line). Analytical solution with $A=2$. The solution is of course independent of $a$. b): corresponding average number of partons per clan, $\bar{n}_{c}\left(y_{c}, W\right)$, as a function of the width of the rapidity interval $y_{c}$ at different maximum allowed virtualities $W=50 \mathrm{GeV}$ (solid line), 100 $\mathrm{GeV}$ (dashed line) and $500 \mathrm{GeV}$ (dotted line). Analytical solution with $A=2$ and $a=1$. 

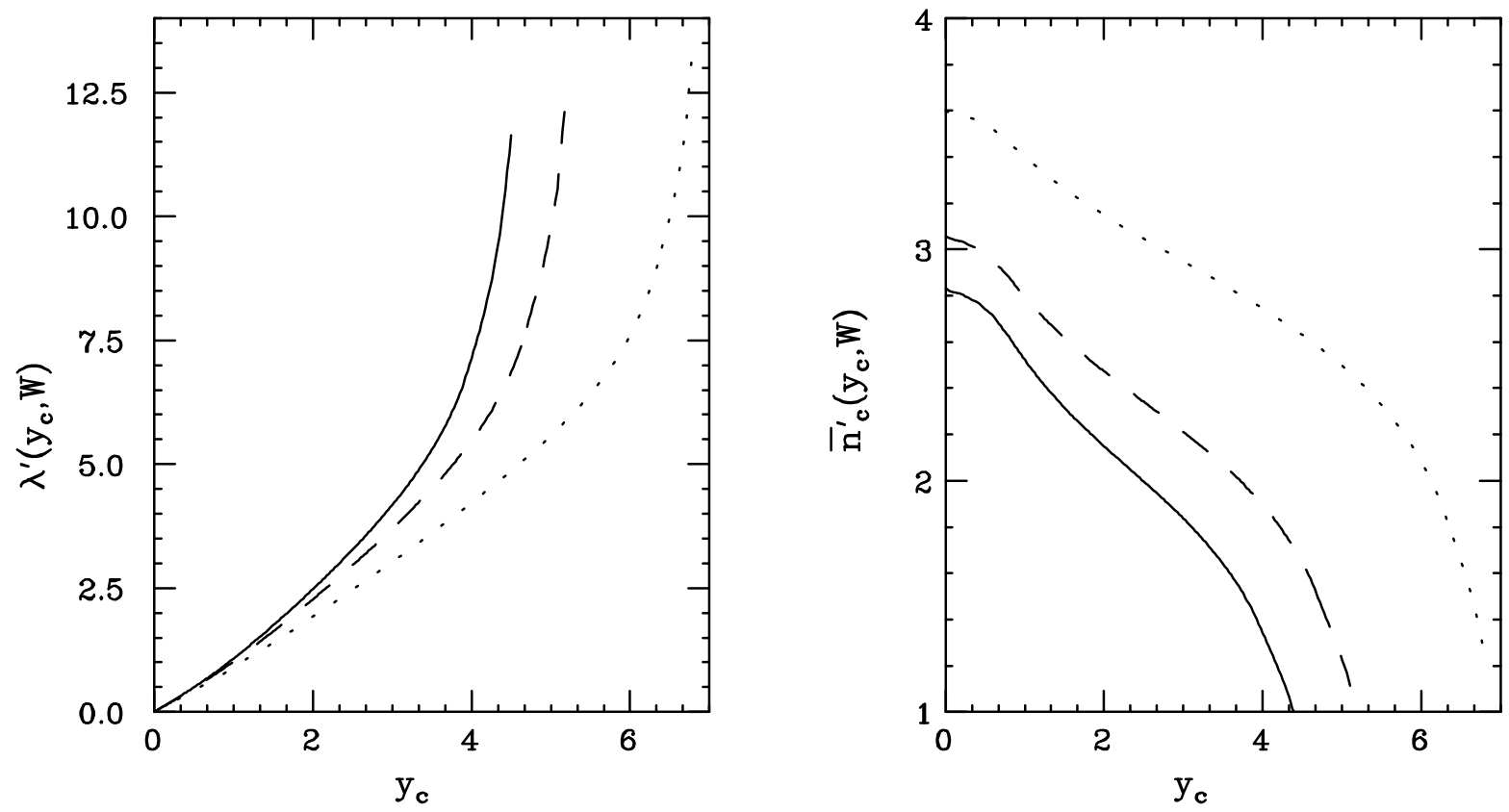

Figure 6: a): average number of clans, $\lambda^{\prime}\left(y_{c}, W\right)$, defined by requiring that the total MD be a CPD as in Eq. (21), as a function of the width of the rapidity interval $y_{c}$ at different maximum allowed virtualities $W=50 \mathrm{GeV}$ (solid line), $100 \mathrm{GeV}$ (dashed line) and $500 \mathrm{GeV}$ (dotted line). Analytical solution with $A$ $=2$. The solution is of course independent of $a$. b): corresponding average number of partons per clan, $\bar{n}_{c}\left(y_{c}, W\right)$, as a function of the width of the rapidity interval $y_{c}$ at different maximum allowed virtualities $W=50 \mathrm{GeV}$ (solid line), $100 \mathrm{GeV}$ (dashed line) and $500 \mathrm{GeV}$ (dotted line). Analytical solution with $A$ $=2$ and $a=1$. 

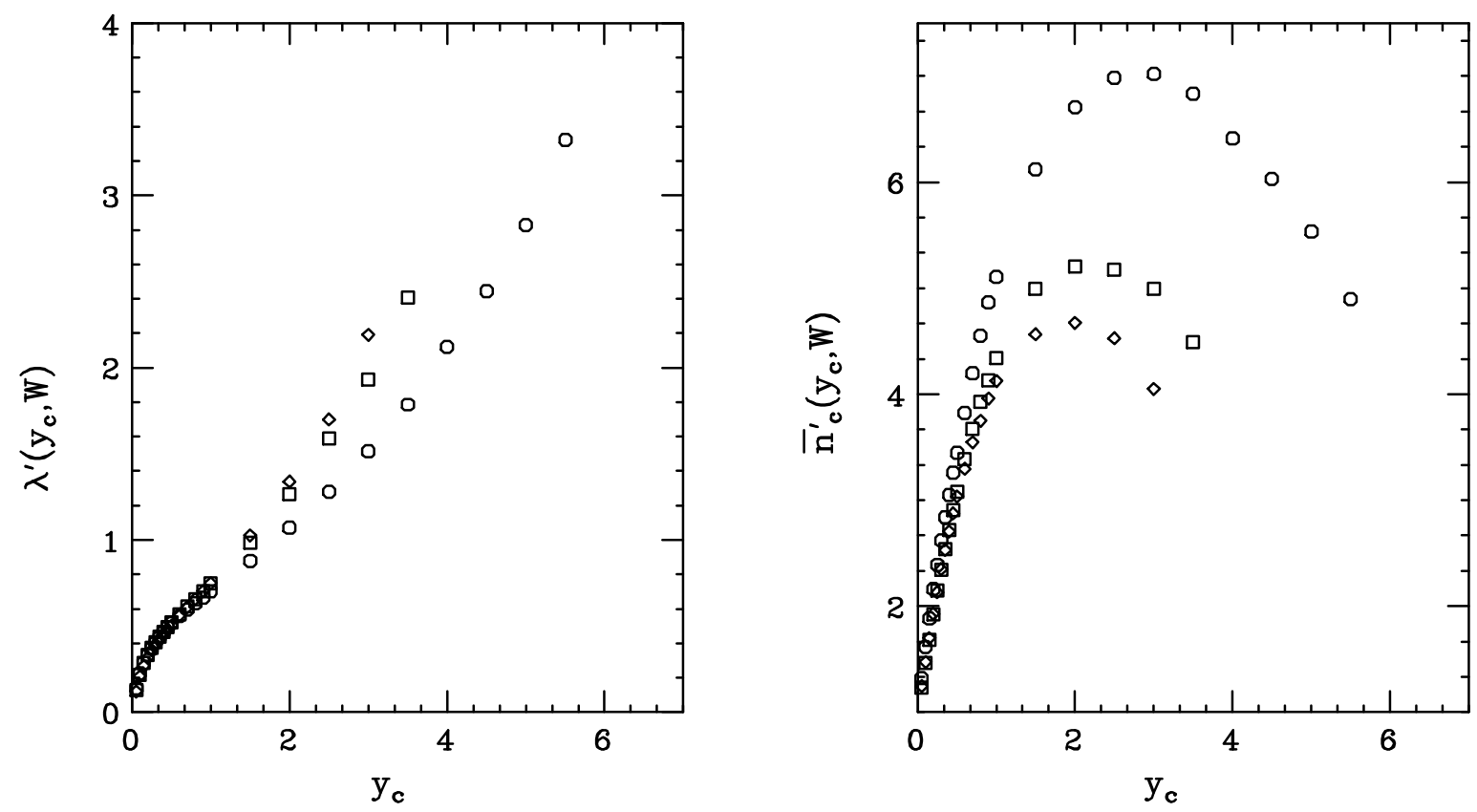

Figure 7: Same as in Figure (6), but Monte Carlo results at different maximum allowed virtualities $W=50 \mathrm{GeV}$ (diamonds), $100 \mathrm{GeV}$ (squares) and $500 \mathrm{GeV}$ (circles). 